\title{
Advances in Single Particle Analysis Data Acquisition
}

\author{
Stan Konings ${ }^{1 *}$, Maarten Kuijper ${ }^{1}$, Jeroen Keizer ${ }^{1}$, Fanis Grollios ${ }^{1}$, Tjerk Spanjer ${ }^{1}$, Peter Tiemeijer ${ }^{1}$ \\ 1. Thermo Fisher Scientific, Materials \& Structural Analysis, Eindhoven, the Netherlands \\ * Corresponding author: stan.konings@thermofisher.com
}

Single particle analysis (SPA) is a cryo-transmission electron microscopy technique which is used to unravel and visualize the 3D structure of individual proteins and protein complexes at near atomic resolution. This is achieved by acquiring typically thousands of low dose images of a thin layer of vitreous ice in which the purified protein particles are embedded preferably in random orientations. Post processing software is used to identify the individual particles in the image, classifying and averaging the particles depending on their specific orientation and finally reconstructing and refining the 3D protein structure to atomic detail. Recent progress in single particle analysis has led to increasingly higher 3D resolutions down to sub $2 \AA$. The key technological advances which have made this possible are the development of electron-counting direct electron detectors, automation of electron-optical setup and data collection and improvements in image processing software [1-4].

The next frontier is to reduce the time to acquire the SPA data set. Nowadays the acquisition of a complete data set can take from one to a few days depending on desired 3D-resolution and sample quality characteristics like number of particles / image, particle distribution and orientation. In this presentation, we discuss our advances in reducing the time to acquire a single particle analysis data set as well as improving the quality of the data.

A first example is the illumination scheme which has been adjusted and optimized for the SPA use case. The Fresnel fringes from the beam-limiting condenser aperture which used to be observed at the outer parts of the illuminated areas is virtually eliminated at typical SPA magnifications (See Figure 1). Apart from better image quality, this fringe free illumination scheme allows for smaller illumination areas i.e. an area which is just slightly larger than the sensitive area of the camera. This enables higher data acquisition throughput since the time penalty for targeting is reduced as more images per target area (typically a 1 to 2 micron-sized hole filled with vitreous ice in a carbon foil) are possible.

In addition, a new and fast acquisition method is implemented in the EPU2.x data acquisition software which makes use of enlarged image shift ranges (up to $10 \mu \mathrm{m}$ ) for targeting the acquisition areas, while keeping the image-shift induced aberrations within negligible level (below $1 \mu \mathrm{m}$ coma). This aberrationfree image-shift method (AFIS) creates another improvement of data acquisition throughput since image shift is much faster than the traditionally used stage shift and moreover does not create any sample drift.

Special attention is given to new direct electron detection cameras that will be integrated in the single particle data-acquisition software. These cameras offer not only a higher image quality, as measured by the detective quantum efficiency (DQE) but also much faster recording speeds.

Moreover, improvements in the screening and data-acquisition software EPU2.x reduce the time needed to select the best sample areas for acquisition, but also eases the setup of the data collection experiment. The EPU software also supports a new data handling application and post-processing platform which allows for direct data quality monitoring and a seamless sample and data connectivity for the user. 


\section{References:}

[1] The Nobel Prize in Chemistry 2017, Jacques Dubochet, Joachim Frank, Richard Henderson.

[2] Nature Methods, Special feature: Method of the Year 2015 (2015).

[3] KR Vinothkumar and R Henderson, Quarterly Reviews of Biophysics (2016).

[4] A Boland, L Chang, D Barford, The potential of cryo-electron microscopy for structure-based drug design, Essays in Biochemistry (2017).
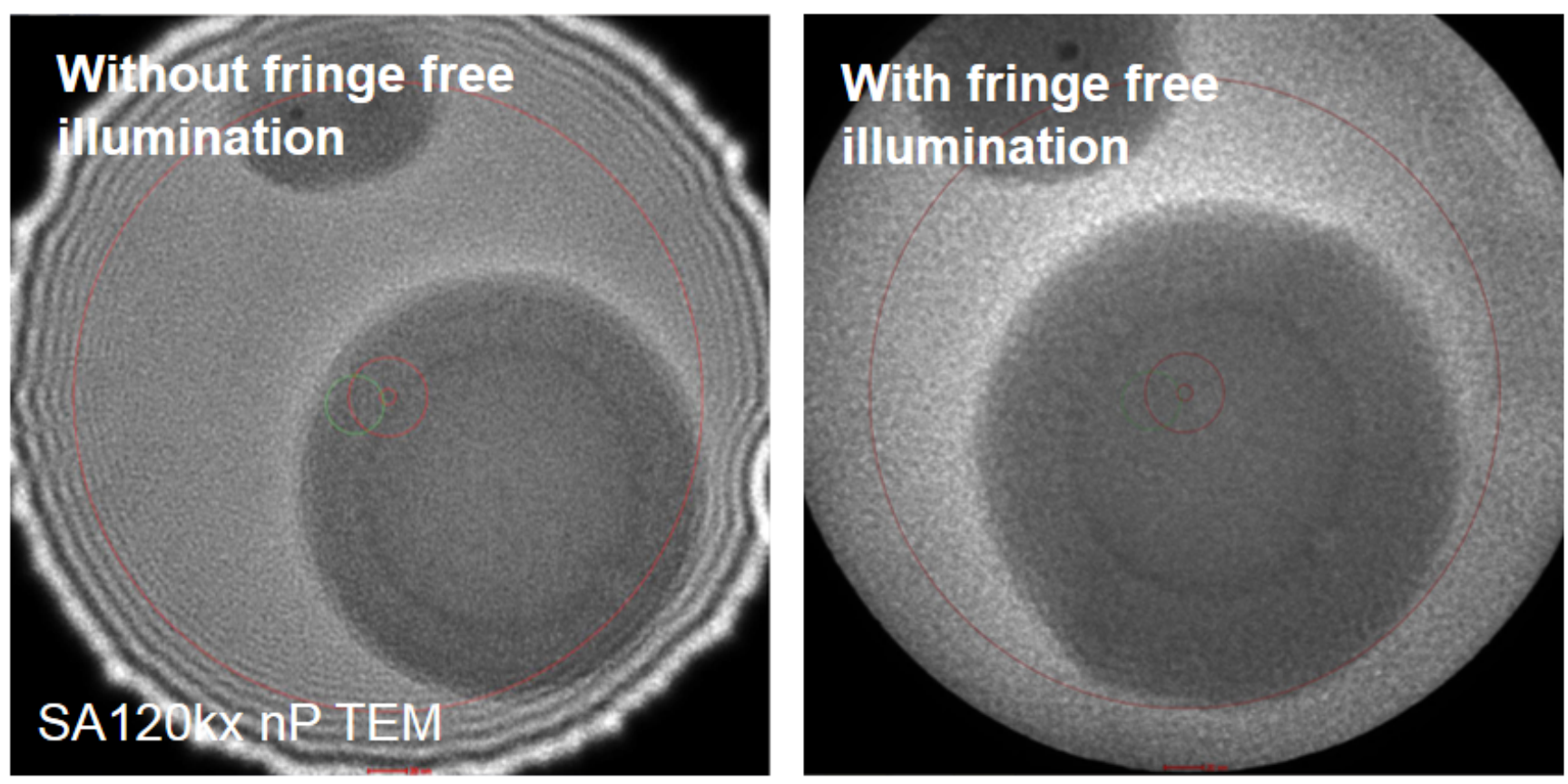

Figure 1. Transmission electron microscopy image taken on Thermo Scientific Krios at a magnification of 120,000x (Left): conventional illumination scheme in which the image is in focus, but diffraction from the beam limiting condenser aperture leads to Fresnel fringes. (Right): new fringe free illumination scheme. The Fresnel fringes are eliminated since the condenser aperture and the sample are in conjugate image planes. 